\title{
A COOPERATIVE LOCALIZATION METHOd BASED ON V2I COMMUNICATION AND DISTANCE INFORMATION IN VEHICULAR NETWORKS
}

\author{
Walaa Afifi ${ }^{1}$, Hesham A. Hefny ${ }^{2}$ and Nagy R. Darwish ${ }^{1}$ \\ ${ }^{1}$ Department of Information Systems and Technology, Faculty of \\ Graduate Studies for Statistical Research, Cairo University, Egypt \\ ${ }^{2}$ Department of Computer Sciences, Faculty of Graduate Studies \\ for Statistical Research, Cairo University, Egypt
}

\begin{abstract}
Relative positions are recent solutions to overcome the limited accuracy of GPS in urban environment. Vehicle positions obtained using V2I communication are more accurate because the known roadside unit (RSU) locations help predict errors in measurements over time. The accuracy of vehicle positions depends more on the number of RSUs; however, the high installation cost limits the use of this approach. It also depends on nonlinear localization nature. They were neglected in several research papers. In these studies, the accumulated errors increased with time due to the linearity localization problem. In the present study, a cooperative localization method based on V2I communication and distance information in vehicular networks is proposed for improving the estimates of vehicles' initial positions. This method assumes that the virtual RSUs based on mobility measurements help reduce installation costs and facilitate in handling fault environments. The extended Kalman filter algorithm is a well-known estimator in nonlinear problem, but it requires well initial vehicle position vector and adaptive noise in measurements. Using the proposed method, vehicles' initial positions can be estimated accurately. The experimental results confirm that the proposed method has superior accuracy than existing methods, giving a root mean square error of approximately $1 \mathrm{~m}$. In addition, it is shown that virtual RSUs can assist in estimating initial positions in fault environments.
\end{abstract}

\section{KEYWORDS}

Cooperative localization, Data fusion algorithms, V2X communication.

\section{INTRODUCTION}

Nowadays, transportation problems, including accidents and traffic jams, affect people's lives in many ways. Intelligent transportation systems (ITSs) can help to overcome such problems by utilizing wireless communication between roadside units (RSUs) and onboard units (OBUs) that are attached to vehicles, or by utilizing wireless communication between vehicles, to provide services to drivers and passengers [1]. However, the multipath effect, the non-line-of-sight conditions, and the presence of obstacles reduce the degree of certainty in the associated measurements and reduce the signal strength. Therefore, range measurement methods are characterized by a large degree of uncertainty, and there is a greater need to adapt parameters such as the path exponent and noise parameters. A limited number of smart sensor nodes, i.e., inertial navigation systems (INSs), which include accelerometers, gyroscopes, compasses, and odometers [2], are attached to vehicles to measure their speeds and directions. The accuracy of such measurements is closely related to the cost of the sensors, which means that the price of 
vehicles is continuously high. The position of a vehicle is a vital element in most (ITSs) and is required to be known so that messages and services can be delivered accurately. GPS systems are a well-known type of absolute positioning system whose accuracy is limited to around 20 to $30 \mathrm{~m}$ because of the non-line-of-sight problem and the presence of obstacles [3, 4]. In [5], the authors enhanced the quality of service provided by the greedy perimeter stateless routing protocol (GPSR). The quality of the GPSR depends on the accuracy of the GPS positions. They used a Kalman filter GPSR (KF-GPSR) to improve the accuracy of the GPS positions. This led to an enhanced packet delivery ratio and reduced overheads, meaning that the KF-GPSR outperformed the standard GPSR protocol. Kinematic modeling or dead reckoning calculates positions based on measurements that are obtained from INSs. The final position within each time interval is updated based on the speed and direction of the vehicle as follows [6]:

$$
\begin{aligned}
& x_{k}=x_{k 0}+\sum_{i=1}^{k} s_{i} \cdot \cos \left(\theta_{i}\right) \\
& y_{k}=y_{k 0}+\sum_{i=1}^{k} s_{i} \cdot \sin \left(\theta_{i}\right) .
\end{aligned}
$$

Here, $\boldsymbol{x}_{\boldsymbol{k}}$ and $\boldsymbol{y}_{\boldsymbol{k}}$ are the coordinates of the vehicle position at the current time $k$; ${ }^{x_{k 0}}$ and $y_{k 0}$ are

the initial coordinates at the initial time $\mathrm{k} 0 ;{ }^{S_{\tilde{i}}}$ is the velocity at the time step $i$; and $\theta_{i}$ is the direction of motion of the vehicle at time $i$. Any inaccuracy in the final position causes errors to accumulate over time. Some researchers have combined two existing techniques to improve the position accuracy. INS and GPS systems are used together to measure positions in urban or tunnel environments. Data fusion algorithms are then used to enhance these measurements. However, this still does not provide a good enough solution over long periods due to the highly dynamic nature of the network topology, inaccuracy initial position, noise in measurements and nonlinearity of the problem $[7,8]$. Cooperative localization is an alternative positioning method that can be used by GPS systems and standalone INS/GPS systems. This method provides relative positions using V2V or V2I communication. It introduces another source of collected measurement to increase the opportunity to obtain well accurate measurements. A V2V communication system can be used to improve the GPS positions, but the noisy environment can still affect the accuracy. A V2I communication system can be used to estimate initial positions, which increases the chance of obtaining more measurements that are accurate and predicting the errors in position [9]. The large distance to RSUs and the number of RSUs have a significant effect on the position accuracy and on attempts to convert the nonlinear problem to a linear one. In [10], the authors used V2I and V2V communication approaches. V2I communication was used to obtain initial vehicle positions by applying the cosine rule. The Kalman filter was then used to increase the accuracy of the initial positions. V2V communication was used to update the vehicle positions based on measurements of relative motion. The use of these methods did not achieve acceptable results because the position accuracy was closely related to the amount of uncertainty in the collected measurements and the errors accumulated over time. Data fusion algorithms such as the Kalman filter and its variations, the particle filter algorithm, least square errors, and the double difference method are used extensively with cooperative localization and object tracking problems to reduce these accumulated errors $[11,12]$. The localization problem is nonlinear by nature. Linearization of this problem can lead to a loss of information and thus have an impact on the accuracy. Data fusion algorithms are more sensitive to initial vehicle position and adaptive noise in measurements. In [13], authors used GPS/INS standalone systems to trace the autonomous vehicle way. This vehicle was equipped with two GPS receivers and INS (i.e. an attempt to get more measurements). The authors used singular value decomposition method to adapt noise covariance matrix in extended Kalman filter algorithms. However, the position accuracy is acceptable to short period time. The degrading performance of GPS and errors to be accumulate over time, the position accuracy was more negative affected. In this paper, a cooperative localization method based on V2I communication and distance information from 
vehicular networks is proposed in which the initial vehicle positions are estimated using a general formula for the intersection of two circles [14]. The initial positions are estimated from RSU locations and distance information. The use of the intersection formula gives more accurate initial positions than updating the positions based on the uncertainty in measurements of the motion as in a kinematic model. Using the intersection method, the vertical distance intersect on road width at point can be determined precisely. Therefore, increased road width help drawing straight line intersect at specified point. In addition, the use of the general formula for intersecting points is not restricted to linear motion models or linear network topology; the formula can also be applied to other models of motion; e.g., models of vehicles moving on square roads and through intersections. Vehicles can also change lanes without any effect on the position accuracy. If an RSU fails, the formula for the intersection of two circles cannot be applied; in this situation, a virtual RSU is used to replace the failed RSU. The position of the virtual RSU is estimated based on measurements of the vehicle's motion and the location of an active RSU. This ensures that the virtual RSU's location is in the same direction relative to the moving vehicle and, therefore, allows continued use of the formula for the intersection of two circles to estimate the intersection point, thus improving the measurement accuracy. Furthermore, the use of virtual RSUs helps to reduce the cost of deploying RSUs on the road. To overcome the synchronization problem, noise in radio measurement and deal with the nonlinear problems, an extended Kalman filter (EKF) can be used to reduce inaccuracies in the distance measurements and reduce the accumulated errors. The experimental results show that the proposed method gives a position accuracy of nearly $1 \mathrm{~m}$, which is better than that obtained using other cooperative localization methods, for which the obtained accuracies were 13, 9, and $6 \mathrm{~m}$ [10, 21, 25], respectively.

The rest of this paper is organized as follows: Section 2 explains the related works with their advantages and disadvantages. Section 3 presents the proposed cooperative localization method. Section 4 provides the experimental results. Section 5 provides discussion and analysis to the experimental results. Section 6 outlines the proposed cooperative localization method based on distance information and explains the directions for future works.

\section{RELATEd STUdies}

Cooperative localization methods can be divided into GPS free localization (i.e., V2I communication) and $\mathrm{V} 2 \mathrm{~V}$ cooperative localization methods. The accuracy of positions determined using V2I communication increases with the number of roadside units, the road width, and the distance to the RSU. If the distance to the RSU is large and the road is wide, the RSU and the vehicle will lie nearly on the same line. Almost all related studies, it is assumed that there is a large number of configured RSUs, or at least four received beacon messages from RSUs are used to estimate vehicle positions. Overhead in communication and uncertainty in the measurements reduce the position accuracy. In [15], the authors used V2I communication and the weighted least square error method to estimate initial positions based on minimizing the errors in the distance measurements. This required at least three messages from individual RSU to be received. Vehicles have a fixed speed; therefore, the distance traveled in a given length of time is fixed. In highly dynamic environments, however, it is difficult to follow the changing rate in spite of various speed and distance, and the effect of the uncertainty in measurements is greater. In addition, localization is an inherently nonlinear problem. In [16], the authors proposed a free GPS localization algorithm based on a single RSU and the use of the angle of arrival (AOA) signal range method. The vehicle's position was estimated using the weighted least square error method. The aim was to minimize the error between the AOA and the estimated angle using equation (6). The authors proposed equation (3) to determine the intersection point, $P_{c}\left(t_{k}\right)$. This required to keep the values of the vehicle's speed, direction, and distance to the RSU correctly updated and thus to determine the direction of the received signal. 
International Journal of Computer Networks \& Communications (IJCNC) Vol.13, No.6, November 2021

$$
P_{c}\left(t_{k}\right)=\left[\begin{array}{c}
x_{c}\left(t_{k}\right)=\frac{u_{t_{k}} m_{t k}}{1+m_{t_{k}}^{2}} \\
y_{c}\left(t_{k}\right)=y_{v}\left(t_{k}\right)+\frac{u_{t_{k}} m_{t_{k}}^{2}}{1+m_{t_{k}}^{2}}-m_{t_{k}} x_{v}\left(t_{k}\right)
\end{array}\right],
$$

Where $m_{t k}$ is the direction in which the vehicle is heading, which is given by

$$
m_{t_{k}}=\frac{v_{y}}{v_{x}}
$$

And

$$
u_{t_{k}}=y_{R}-y_{v}\left(t_{k}\right)+m_{t k} X_{v}\left(t_{k}\right)+\frac{x_{R}}{m_{t k}}
$$

$u_{t_{\mathrm{k}}}$ is the assumed difference between vehicle's $\mathrm{x}$ - coordinate and the RSU's $\mathrm{x}$ - coordinate. It is measured from difference in y coordinates that will be shared with point that is determined by vehicle's mobility parameters. $x_{R}$ and $y_{R}$ are the coordinates of the RSU, and $\mathrm{x}_{\mathrm{v}}\left(\mathrm{t}_{\mathrm{k}}\right)$ and $\mathrm{y}_{\mathrm{v}}\left(\mathrm{t}_{\mathrm{k}}\right)$ are the vehicle's coordinates at time $t_{k}$. The calculated angle $\theta_{k}$ gives the estimated direction of the received signal from the RSU without using multiple smart antennas. $\theta_{k}$ was estimated as

$$
\theta_{k}=\arccos \left(\frac{\left\|p v\left(t_{k}\right)-p c\left(t_{k}\right)\right\|}{\left\|p v\left(t_{k}\right)-p_{R}\right\|}\right)
$$

Each vehicle was equipped with a multiple smart antennas to estimate the different values of the AOA. After this, the MUSIC algorithm was used to minimize the signal noise and obtain the most appropriate value of the AOA [17]. The difference between the AOA and the estimated angle $\theta_{k}$ was minimized using the least square error methods to find the position of the vehicle. The AOA range method is more robust and produces smaller measurements errors; however, the errors can still increase over time and lead to greater uncertainty in the measurements. In [18] the authors proposed an RSU/INS-aided localization system in which each vehicle receives the distance measurements as messages from RSU in one queue. Second queue is used to provide new position estimates based on the INS system. Each vehicle receives at least four messages. Vehicle estimate their initial positions using the least squares method to minimize the errors in the distance and position. A Kalman filter is then applied to improve the position estimates, and the position accuracy is closely related to the accuracy of the initial position. Again, errors accumulate over time because of the nonlinear nature of the problem. In [19], the authors proposed an RSU-based localization schema method. In this method, the vehicle receives at least two messages from each of two RSUs, one on each side of the road, and the vehicle position is determined as being the point where the ranges of the two transmissions intersect. The choice of whether to use the forward or backward intersection point is determined by the direction of the received signal. In the case of failure of one of the RSUs, a second-order equation is used to find the position based on the distance to the currently active RSU, the previous distance, and the previous position of the target vehicle. The point intersection method proved to be more effective in linear vehicle mobility model and high certainty degree of distance measurement. The authors assumed that large numbers of RSUs were placed on both roadsides. As the distance between the RSU's is equal to the transmission range, the cost of this set-up is high. In addition, errors can accumulate as result of the failure of RSUs and because data fusion is not included in this 
method. In [20], the authors used different sources of information to localize the vehicle position: TOA measurements, INS system and map data. Kinematic model parameters were obtained by inertial navigation frame that attached to a vehicle. The INS's mobility measurement was adapted by TOA measurements upon receiving the messages from single RSU (i.e. position was updated by distance measurement). These measurements were fused to EKF algorithm. After that, the position was bounded to selected road boundary. The accuracy of selected road segment or line segment method depended on the accuracy of digital map data. It was known that the accuracy of EKF depended on the initial position and noise covariance matrix. Different sources of measurements had limited accuracy over time. Accumulated errors increased over passed time.

Cooperative localization based on $\mathrm{V} 2 \mathrm{~V}$ communication solves the problem of having to deploy a large number of RSUs and considers another source of exchanging motion measurements. It also takes into account the multipath effect, the signal noise, obstacles, and the density of the network. The position accuracy increases as the number of neighboring vehicles moving in the same direction as the target vehicle increases. In all related studies, data fusion algorithms have been used to handle the nonlinearity problem and signal noise. However, the acceptable position accuracy levels are not enough. There is a direct relation between the uncertainty in measurement and position accuracy. In [21], the authors used GPS/INS systems to obtain the initial positions. The EKF was then used to linearize the distance function and the polar coordinates that were estimated by the kinematic model; this also reduced the accumulated error. The EKF is closely related to the initial parameters and adjusted noise in measurement and process. It is known that the performance of GPSs in urban environment is limited, and INS system is benefit to small period. The uncertainty in the measurements is produced by the range measurements techniques. Therefore, the position accuracy increases as the number of neighbors moving in the same direction increases. This is difficult to achieve in the case of highways and high-error urban environments. In [22], the authors introduced the inter-vehicle communication-assisted localization (IVCAL) algorithm. This algorithm uses more than one data fusion algorithm to reduce the multipath effect and excludes the neighboring measurements that are more seriously affected by the multipath effect; a neural network model is used to determine which neighbors are most affected. The Nelder and Mead algorithm and the Kalman filter are used together to estimate the final position and to reduce errors in measurement. The IVCAL requires a lot of computation time, which means that, it is not suitable for application in safety-critical situations. In [23] the authors proposed a constrained weighting scheme named inter-vehicle communication-assisted localization (CWS-IVCAL), which is an extension of the IVCAL algorithm. This algorithm uses the weight factor to measure the degree of certainty in the distance measurements, and the weighted centroid method is used to determine the final position. The use of the weight factor helps to improve the accuracy of the estimated position. The CWS-IVCAL algorithm outperformed the IVCAL in urban areas, but the position accuracy was more seriously affected by distance errors. In [24], the use of the vehicle location improved algorithm (VLOCI) was proposed. In this algorithm, a weight factor is used to give the nearest neighbor more weight. The weight is calculated as the inverse of the distance or as the exponential function of the distance parameter, and the position is estimated by the weighted centroid method. In this paper, the authors dealt with localization as a linear problem but used an unrealistic static network. In [25], a cooperative vehicle localization improvement using distance information (COVALID) algorithm was proposed. This algorithm is an improved version of the VANET location improved algorithm (VLOCI) and uses the weighted centroid method to estimate the final position; the target vehicle assigns a weight to each neighbor based on the measured distance. The use of both a GPS and INS is required to obtain the initial position, and the difference in the distances measured by the two systems is determined. The rules for similar triangles are then used to search for neighbors that lie on the same line or have a linear relationship with the target vehicle. The position determined by the GPS is then updated by applying the kinematic equations using the difference between the distances measured by the INS and GPS. The COVALID algorithm was 
found to reduce the error in the locations determined by the GPS by 63\%; this is still not good enough for determining the linear relationship between neighboring vehicles, particularly in the case of highways. The uncertainty in the distance between neighbors also affects the degree of similarity. They give constant weight for each neighbor based on different distance ranges. Constant weights do not describe well neighbors in uncertainty in measurements and therefore they may lead to coordinate system outside network area. In [26], the authors proposed the use of a localization algorithm to solve the GPS non-line-of-sight problem. In this study, errors in measurements were minimized by weighted each vehicle neighbor based on received signal strength (RSSI) and signal to noise ratio (SINR).GPS, RSSI and SINR measurements were fused together to EKF algorithm enhance the vehicle position. Therefore, the position accuracy were related with number of neighbors and certainty degree in measurements.

\section{The Proposed Cooperative Localization Method}

The proposed cooperative localization method is based on V2I communication and distance information. It aims to remove the problems encountered when installs a large number of RSUs and provides continued estimates of vehicle positions in error environments. In addition, the nonlinear localization problem is dealt with, and the improved initial states produce better results than those obtained using the EKF. The proposed method is based on the idea of estimating virtual RSUs. Inactive RSUs are substituted by virtual ones so that continuous estimates of vehicle positions can be provided to solve the problem of having a large number of RSUs. The initial positions are determined accurately using mathematical equations. The general equation for the intersection of two circles is a powerful method of calculating expected vehicle positions based on distances and the fixed locations of RSUs [14]. The use of the EKF also reduces the uncertainty in the measurements and permits linearization of the distance function. The proposed method consists of the following steps.

\subsection{V2I Communication}

As shown in figure 1, V2I communication is first used to estimate the initial position based on the intersection method. There are two RSUs - one on each side of the road. The transmission range of the RSUs is twice that of the vehicles'. Each RSU sends out messages periodically. These messages include the position of each RSU, its identification number and the time of sending. To avoid packet loss, the interval between messages can be adjusted by setting a jitter time randomly between 0 and 1 :

$$
\text { Time interval }=\text { time interval }+ \text { jitter time }
$$

Therefore, the risk of packet loss can be reduced and the number of received messages can be increased. In figure 1, vehicle $i$ receives messages from both RSUs and the identification number, distance, and time of receipt for each packet.

\subsection{Estimating the Vehicle Position}

After receiving the messages from the RSUs, the vehicle estimates its own position using the intersection method [14]. The two RSUs are located at fixed position and have the same $y$ coordinates but different $x$-coordinates, the difference between the $x$-coordinates being equal to the road width. Each vehicle estimates the distance between itself and the RSUs based on the time of arrival or receipt of the first signal:

$$
r_{i j}=T_{i j}, c+e
$$


Where $r_{i j}$ is the estimated distance between vehicle $i$ and $\operatorname{RSU} j(j=1,2), c$ is speed of light $(3 \times$ $10^{8} \mathrm{~m} / \mathrm{s}$ ), and $T_{i j}$ is the time at which vehicle $i$ receives a message from RSU $j$. These distances represent the transmission range of the vehicle. The distance between the two RSUs is equal to the width of the road, $R_{12}$. This must be less than the sum of the distances between the vehicle and the two RSUs, $r_{i 1}+r_{i 2}$, and greater than the absolute difference between them. This condition means that the vehicle receives messages from both RSUs and is, therefore, within the transmission range of both RSUs:

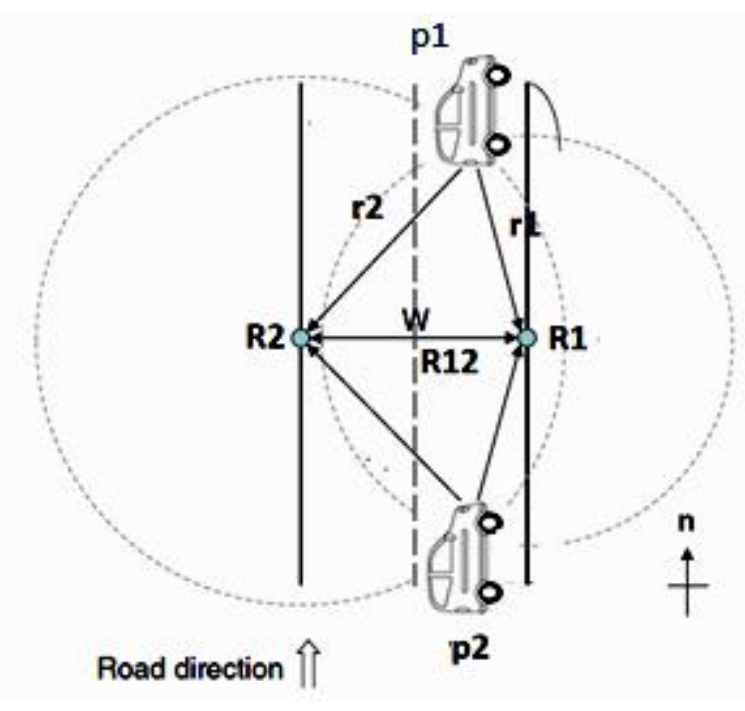

Figure 1. Points of intersection between the transmission ranges of two different RSUs

$$
r_{i 1}+r_{i 2}>R_{12}>\left|r_{i 1}-r_{i 2}\right|
$$

It is equally likely that the vehicle is located at either of the two possible range intersection points, whose coordinates are given by

$$
x_{p j}=x_{R 1}+\lambda_{i} x_{d} \mp \epsilon_{i} y_{d}
$$

And

$$
y_{p j}=y_{R 1}+\lambda_{i} y_{d} \pm \epsilon_{i} x_{d}
$$

Where $j(j=1,2)$ refers to the two possible points of intersection, $\mathrm{P} 1$ and $\mathrm{P} 2$. The coordinates of RSU R1 are $x_{R I}$ and $y_{R I} ; \mathrm{x}_{\mathrm{d}}$ and $y_{\mathrm{d}}$ represent the difference between the $x$ and $y$ coordinates of the two RSUs, respectively:

$$
\begin{aligned}
& x_{d}=x_{R 1}-x_{R 2} \\
& y_{\mathrm{d}}=\mathrm{y}_{\mathrm{R} 1}-\mathrm{y}_{\mathrm{R} 2} \\
& \lambda_{i}=\frac{r_{i 1}^{2}-r_{i 2}^{2}+R_{12}^{2}}{2 R_{12}^{2}}
\end{aligned}
$$




$$
\epsilon_{i}=\sqrt{\left|\frac{r_{i 1}^{2}}{R_{12}^{2}}-\lambda_{i}^{2}\right|}
$$

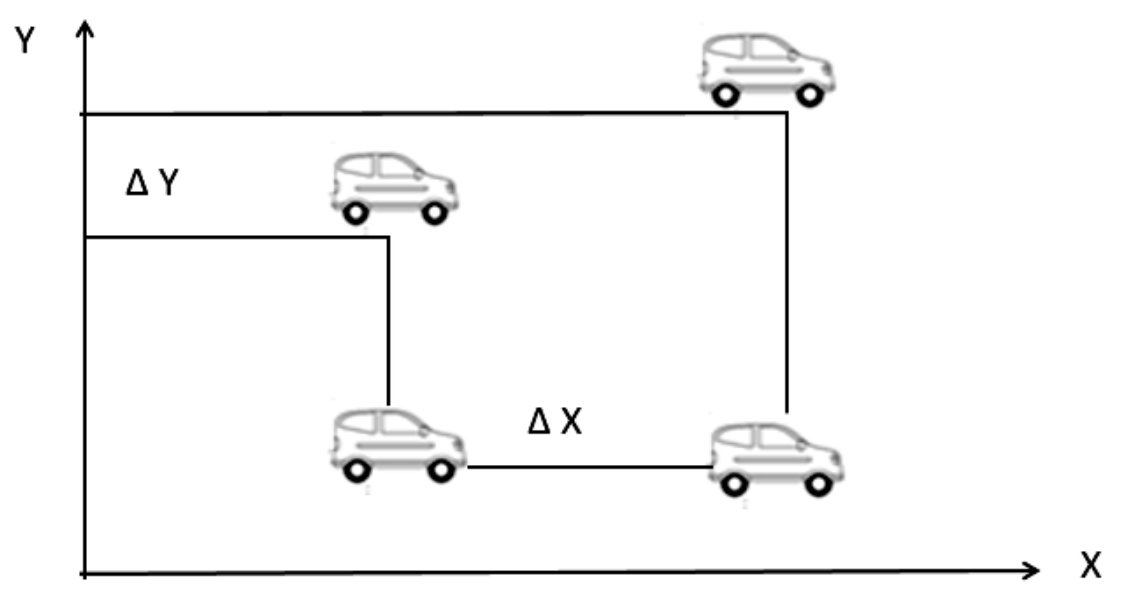

Figure 2. The rate of change in two dimensions

Where $\lambda_{i}$ is equal to $\Delta x$ in figure 2, which illustrates the use of the cosine rule. The difference between the distances is given by $\Delta y$, and adding the distance between the RSUs, $R_{12}^{2}$, to measure $\Delta x$ coordinate that equal to road width or cosine rule. $\epsilon_{i}$ is equal to the value of $\Delta y$. It finds the square root for the difference between distances rates and $\lambda_{i}$ to measure the $\Delta y$ accordance to the distance. The vehicle $i$ then decides which of points $\mathrm{P} 1$ or P2 it is located that is based on the directions

$$
\theta_{i 1}=\cos ^{-1} \frac{r_{i 1}^{2}+R_{12}^{2}-r_{i 2}^{2}}{2 \cdot r_{i 1} \cdot R_{12}}
$$

And

$$
\theta_{i 2}=\cos ^{-1} \frac{r_{i 2}^{2}+R_{12}^{2}-r_{i 1}^{2}}{2 \cdot r_{i 2} \cdot R_{12}}
$$

Here $\theta_{i 1}$, and $\theta_{i 2}$ represent the direction or heading of the vehicle. Vehicle $i$ chooses P1 as its location if $\theta_{i 1}$ is less than $\theta_{i 2}$; it chooses $\mathrm{P} 2$ if $\theta_{i 2}$ is less than $\theta_{i 1}$. In this case, it is not necessary for the distance to the RSUs to be large to guarantee the linearity of the problem because the EKF linearizes the nonlinear problem.

\subsection{Failure of an RSU}

Figure 3 illustrates the scenario where one of the RSUs has failed. The failed RSU then becomes a virtual RSU, and its coordinates are estimated using data about the vehicle's motion and the location of the active RSU upon receipt of messages from the active RSU. The vehicle position is then estimated by intersection method. To satisfy the intersection condition, the $y$-coordinate of 
the virtual RSU must be equal to the $y$-coordinate of the active RSU. The $x$ coordinate of the virtual RSU depends on the changes in the $x$ coordinate with distance and velocity:

$$
\begin{gathered}
m_{t+1}=\frac{v_{y}(t+1)}{v_{x}(t+1)} \\
u_{t+1}=d_{R i}(t+1)-d_{R i}(t)+m \cdot x_{i}(t)+\frac{X_{R}}{m} \\
a_{t+1}=1+m_{t+1}^{2}
\end{gathered}
$$

And

$$
P V_{r s u}=\left[\begin{array}{c}
p v_{x}=\frac{u_{t+1} m_{t+1}}{a_{t+1}} \\
p v_{y}=y_{R}
\end{array}\right]
$$

Here $v_{x}(t+1), v_{y}(t+1)$ are the components of the vehicle's velocity at time $t+1 . m_{t+1}$ measures the mile in velocity vector; i.e., it gives the vehicle's heading. If $m_{t+1}$ is large, this means that there has been a large change in the $y$-coordinate and a small change in the $x$ coordinate since the last measurement. The $x$ coordinate of the virtual RSU increases slightly compared to the $x$ coordinate of the active RSU; the virtual road width is small. $u_{t+1}$ helps to determine point that spaced from RSU's $x$ - coordinate in any direction and specified distance according to last vehicle position. The difference in distances from time $(t)$ to $(t+1)$ is positive or negative to determine direction of vehicle i.e. forward or backward direction. This difference is added to estimate changing in vehicle's $\mathrm{x}$ coordinate and RSU's $\mathrm{x}$ coordinate to measure horizontal distance from RSU. The value of $\left(a_{t+1}\right)$ is equal to one plus the square of $m_{t+1}$ to increase the horizontal dimension to get more precise location. This allows for an increased road width but it remains within the network boundary. Once the coordinates of the virtual RSU have been estimated, the next step is to determine the intersection point as described in subsection 3.2. Following this, the EKF is applied, as described in the next subsection.
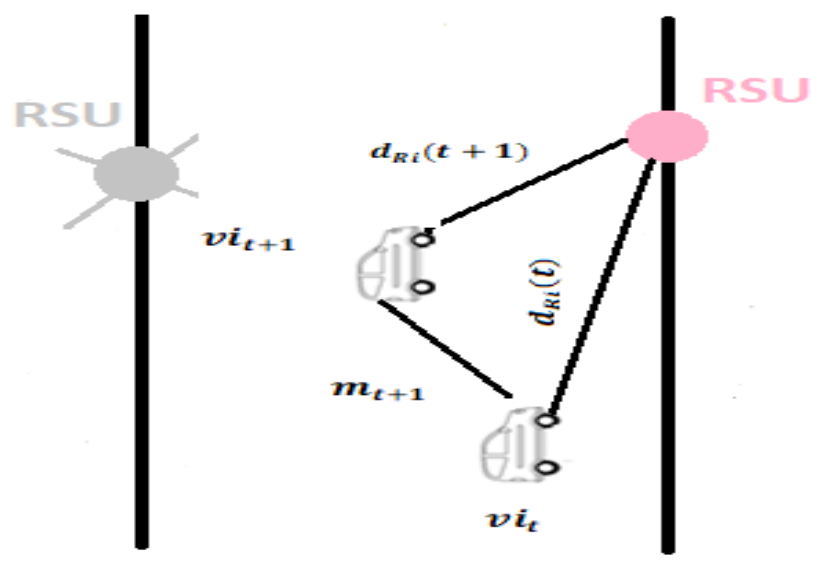

Figure 3. Illustration of a scenario where one RSU has failed 


\subsection{Application of the Extended Kalman Filter (EKF)}

The EKF is the best method of improving the accuracy of determined positions and solving nonlinear localization problems [27, 12]. The EKF works by linearizing a nonlinear function using the partial derivatives. It includes the main steps of the standard Kalman filter-i.e., the time update or prediction step and the measurement update or correction step. The prediction step is responsible for estimating the initial state based on the current state and the covariance noise. The updated measurement step corrects the initial state based on new measurements and the Kalman gain to obtain the final state, which then becomes the new initial state in the next prediction step. These steps are repeated until a specified number of iterations or specified tolerance error threshold is reached. In this paper, the emphasis is on minimizing the error in the distance; i.e., the Euclidean distance between the RSU and the vehicle.

The prediction step makes use of the initial state vector and the initial covariance matrix. The previous state vector, $X_{k-1}$, is equal to the intersection point and the associated measurement noise, $E_{I N S}$, as determined from difference between INS measurements to position at time $k$ - 1 :

$$
X_{k}=X_{k-1}+E_{\text {INS }}
$$

The initial covariance matrix $P_{k}$ represents the error in the position vector. $p_{k-1}$ is the initial covariance matrix, and

$$
P_{k}=p_{k-1}+Q_{k-1}
$$

,$Q_{k-1}$ is the noise covariance square matrix that follows a Gaussian distribution with a mean of 0 and variance $\sigma^{2}$.

The measurement update or correction step starts with the linearization of the function describing the Euclidean distance function between the vehicle and the two RSUs. This linearization is performed using a Taylor series expansion; the function $f(x, y)$ is partially differentiated with respect to its parameters:

$$
\mathrm{f}(\mathrm{x}, \mathrm{y})=\sqrt{\left(x_{k-1}-x_{R x \mathrm{i}}\right)^{2}+\left(y_{k-1}-y_{R y \mathrm{i}}\right)^{2}} \quad i=1,2
$$

Here $x_{k-1}, y_{k-1}$ are the vehicle coordinates, and $x_{R x i}, y_{k y i}$ are the RSU coordinates. The partial derivatives can be expressed as a Jacobian matrix $H$ with $N \times 2$ dimensions, where $N$ is the number of RSUs:

$$
\begin{aligned}
\partial f\left(x_{k-1}, y_{k-1}\right) & =f\left(x_{k-1}, y_{k-1}\right)+\frac{\partial f\left(x_{k-1}, y_{k-1}\right)}{\partial x_{k-1}} \cdot\left(x_{k-1}-x_{R 1,2}\right) \\
& +\frac{\partial f\left(x_{k-1}, y_{k-1}\right)}{\partial y_{k-1}} \cdot\left(y_{k-1}-y_{R 1,2}\right)
\end{aligned}
$$


$H=$

$$
\left[\begin{array}{cc}
\frac{\left(x_{k-1}-x_{R 1}\right)}{\sqrt{\left(x_{k-1}-x_{R 1}\right)^{2}+\left(y_{k-1}-y_{R 1}\right)^{2}}} & \frac{\left(y_{k-1}-y_{R 1}\right)}{\sqrt{\left(x_{k-1}-x_{R 1}\right)^{2}+\left(y_{k-1}-y_{R 1}\right)^{2}}} \\
\frac{\left(y_{k-1}-y_{R 2}\right)}{\left(x_{k-1}-x_{R 2}\right)^{2}+\left(y_{k-1}-y_{R 2}\right)^{2}} & \frac{\left(x_{k-1}-x_{R 2}\right)}{\left(x_{k-1}-x_{R 2}\right)^{2}+\left(y_{k-1}-y_{R 2}\right)^{2}}
\end{array}\right]
$$

The Kalman gain,K, represents the relation between the predicted state and the errors in the measurements given by the two-dimensional matrix $R$ :

$$
\mathrm{K}=p_{k} H^{T}\left(H p_{k} H^{T}+R\right)^{-1}
$$

Where $p_{k}$ is the initial covariance matrix, $H$ is the Jacobian matrix, and $R$ is the noise covariance matrix for the measurements. The noise equals the difference between the two estimated positions and followed position estimated by the INS measurements (1), (2) plus an error $E$ that follows a Gaussian distribution with mean 0 and variance $\sigma^{2}$ :

$$
R=\left[\begin{array}{c}
p_{I N S}-P_{e s t}+E \\
\cdots \\
p_{I N S}-P_{e s t}+E
\end{array}\right] .
$$

If the errors in measurements given by $R$ are small, this means that the Kalman gain $(K)$ has a high value and the initial state will be adjusted primarily by using the updated measurements. If $R$ is high, the value of $K$ is small. The initial state will then be adjusted primarily by using the predicted covariance matrix. If the values in the predicted covariance matrix $P$ are small, then the errors in the measurements can mostly be ignored and the predicted state will be close to the true position. The final position is obtained by updating the current state, $x_{k}$, to the posteriori state vector, $X_{k+1}$, as follows:

$$
X_{k+1}=x_{k}+K\left(Y-A x_{k}\right)
$$

Where $\mathrm{A}$ is the square identity transition matrix, $K$ is the Kalman gain, $\mathrm{Y}$ is new INS's measurement and $\left(Y-A x_{k}\right)$ represents the error in the measurements. The final step is the updating of current covariance matrix $P_{k}$ to give posteriori covariance matrix $P_{k+1}$ :

$$
P_{k+1}=(\mathrm{I}-K H) p_{k}
$$

Where $I$ is the identity matrix, $H$ is the transition matrix, and $p_{k}$ is the previous predicted covariance matrix at time $k . X_{k+1}, P_{k+1}$ gives the current predicted state and covariance matrix, respectively at time $\mathrm{k}+1$ that will become priori states in prediction step. The prediction and update steps are repeated every time the vehicle's position continues to be updated with each new measurement. In the section describing the simulations that follows, each vehicle continues to estimate its position each time it receives messages so that it can get accurate measurements and make an accurate estimate of its position. The squared errors are estimated at each time, the position estimates are repeated, and the averages of all the results are taken. 


\section{Simulated SCEnARIOS}

In this section, the accuracy to which vehicle positions can be determined using the proposed cooperative localization method based on V2I communication and distance information is assessed and a comparison made with the methods described in other related papers [10, 21, 25]. The differences in these methods are mainly concerned with the different ways in which the initial positions are improved using data fusion algorithms. In [10], the authors used the cosine rule method to obtain the initial positions. To apply the cosine rule, it is necessary to use different values of $y$ coordinates for the two RSUs and for the values of the $x$-coordinates to be similar because the resulting acute angle and large distance to the RSUs gives a better accuracy for the estimated position. The Kalman filter is then used to improve the initial vehicle position and solve the problem of the accumulated errors. In [21], the authors relied on GPS/INS systems to obtain the initial positions and subsequently update them. It is well known that the accuracy of GPS systems is limited in urban environments due to the non-line-of-sight problem and the multipath effect $[3,4]$. In addition, the errors associated with INS systems can accumulate with time. The initial positions were thus improved by applying an EKF based on distance measurements that were obtained by V2V communication. In [25], the authors used a weighted centroid method to improve the positions obtained using the GPS and INS, and weight factors were used to describe the degree of closeness between the target vehicle and its neighbors. The authors then divided the measured distances into categories, using a fixed weight for each category. The rules for similar triangles were used to find the neighbor for which the relationship between the target vehicle and its neighbor was the nearest to being linear. These results were also improved by applying an EKF algorithm. The performance of the previous methods are evaluated by applying them to two different scenarios. The first of these concerned a one-way road in the city of Enlargen where vehicles entered from the same point. The second scenario was more complex and concerned different roads in the city of KarradaIn where vehicles entered from different points; the vehicles thus approached from different directions and had different road coordinates. Each road consisted of a single lane and single direction. For the second scenario, comparisons were made between the proposed method and the localization methods presented in $[21,25]$ to test the dependency of the EKF algorithm on the accuracy of the initial position; adjustments to the noise were also made during the measurement process. The performance metric used to measure the position accuracy was the root mean square error (RMSE).

The RMSE measures the difference between a real position, $p_{i}$, and an estimated position, $p_{i}{ }^{\wedge}$ :

$$
R M S E=\sqrt{\frac{\sum_{i=1}^{N}\left(p_{i}-p_{i}^{\wedge}\right)}{N}} .
$$

OMNET $5++$ is an open source $\mathrm{C}++$ discrete event network simulator. It applies the IEEE 802.11p standard for vehicular networks [28] and consists of different frameworks that allow the easy development of ad hoc routing protocols such as VEINS [29]. The VEINS framework is a specialized framework used for translating road traffic networks that are described in XML files. These road traffic networks are generated by a SUMO framework [30, 31], which is a bidirection coupled program to network simulator. It is a microscopic car-forwarding model, and consists of a set of programs that enable the creation of road traffic networks. VEINS can be coupled with SUMO to translate actual road traffic into vehicle speeds, directions, positions, road identifiers, traffic lights, intersections or junction nodes, etc. to allow easy implementation of routing protocols. In other words, VEINS provides a client-server model that links OMNET 5++ and SUMO. Table 1 summarizes the simulation parameters that were used in this study. Vehicular networks follow the IEEE 802.11p standard that is used to support ITSs, and a lognormal shadowing propagation model with a path exponent of 1.5 is used. The noise in the 
scenarios that were investigated had a Gaussian distribution with a mean of 0 and variance $\sigma^{2}$. A new vehicle entered the roads every $1 \mathrm{~s}$. The default mobility model used in SUMO applications is the Krauss model, which is a car-following mobility model that describes the behavior of a following vehicle based on the behavior of the vehicle in front. The simulation time used was 60 $\mathrm{s}$, and an average of the results was taken at the end of each simulation. Each scenario was run five times, and averages were again taken.

Table 1. Simulation parameters

\begin{tabular}{|c|c|}
\hline Parameter & Value \\
\hline IEEE standard & $802.11 \mathrm{p}$ standard \\
\hline Number of vehicles & 1 vehicle/s \\
\hline Propagation model & Lognormal shadowing \\
\hline Mobility model & Krauss \\
\hline Pause time & $1 \mathrm{~s}$ \\
\hline Acceleration & $2.6 \mathrm{~m} / \mathrm{s}^{2}$ \\
\hline
\end{tabular}

\subsection{Scenario 1}

In the Enlargen scenario, RSUs were placed on each side of the road at coordinates $(0,1500)$ and $(12,1500)$ to test the proposed localization method. They were placed at $(600,10)$ and $(400,800)$ in the test of the cooperative localization method based on V2I communication, KF, and V2V communication (V2I $+\mathrm{KF}+\mathrm{V} 2 \mathrm{~V}$ ) described in [10]. The network area used was equal to $2500 \mathrm{~m}$ $\times 2500 \mathrm{~m}$. Figure 4 shows the values of the RMSE at six nodes obtained during the simulation. The position errors obtained using the proposed method is the smallest at approximately $1 \mathrm{~m}$ for all six nodes. In the test of the V2I + KF + V2V method, the authors used V2I communication, the cosine rule, $\mathrm{V} 2 \mathrm{~V}$ communication, and the Kalman filter to improve the initial position estimates and to reduce the accumulated errors. However, the accuracy is nearly $13 \mathrm{~m}$, this method gave the least accuracy among the methods tested. The accumulated errors increase with time and the nature of localization is nonlinear. The KF algorithm is more sensitive to the initial state, the noise, and the measurement covariance matrix. In addition, because of lane changes, vehicle position is estimated by cosine rule, these angles may have to be calculated using the sine rule to get better initial position state. One advantage of the proposed method is that a general formula for the intersection point is used to make good estimates of initial positions; these are then improved by applying the EKF algorithm. In [25], which describes the use of the centroid + EKF method, the authors used the weighted centroid method to estimate positions and the rules for similar triangles to find the neighbor that lies on the same line; however, the results here show that this produces higher errors than the GPS/INS +EKF method [21]. Again, this is because of the errors that accumulate during the simulation, the way of obtained initial positions and the use of constant weight factors. In [20,24], two methods use the same source of measurement GPS and INS. The weight factors did not differeinate well between neighbors. Figure 5 shows the position accuracies obtained using the different methods at different velocities ranging from 70 $\mathrm{km} / \mathrm{h}$ to $120 \mathrm{~km} / \mathrm{h}$. The errors for the proposed method are the smallest and are less affected by the speed. This demonstrates the benefit of using a virtual RSU to make continued estimates of vehicle positions within the error environment and the use of the general formula for the intersection of two circles to effectively increase with the road width. In addition, the nonlinear relationships are handled by the EKF without much loss of information. For the V2I + KF + V2V cooperative localization method, the greatest position errors occur at a speed $90 \mathrm{~km} / \mathrm{h}$; for other speeds, the errors are almost as large. It thus appears that the use of V2V communication does not reduce the accumulated errors because increasing uncertainty measurements upon high dynamic network mobility and the unsuitability of the cosine rule. The values of the RMSE for the 
International Journal of Computer Networks \& Communications (IJCNC) Vol.13, No.6, November 2021

centroid +EKF and the GPS/INS + EKF methods are similar at a speed of $90 \mathrm{~km} / \mathrm{h}$ and less different at other velocities because we assume less error in distance measurement. In addition, the probability of finding neighbors that is moving in the same direction increases. At the end of first scenario, the ways for obtained initial position play significant effect during the simulation run. The effect of data fusion algorithms are limited due to initial state and noise covariance matrix.

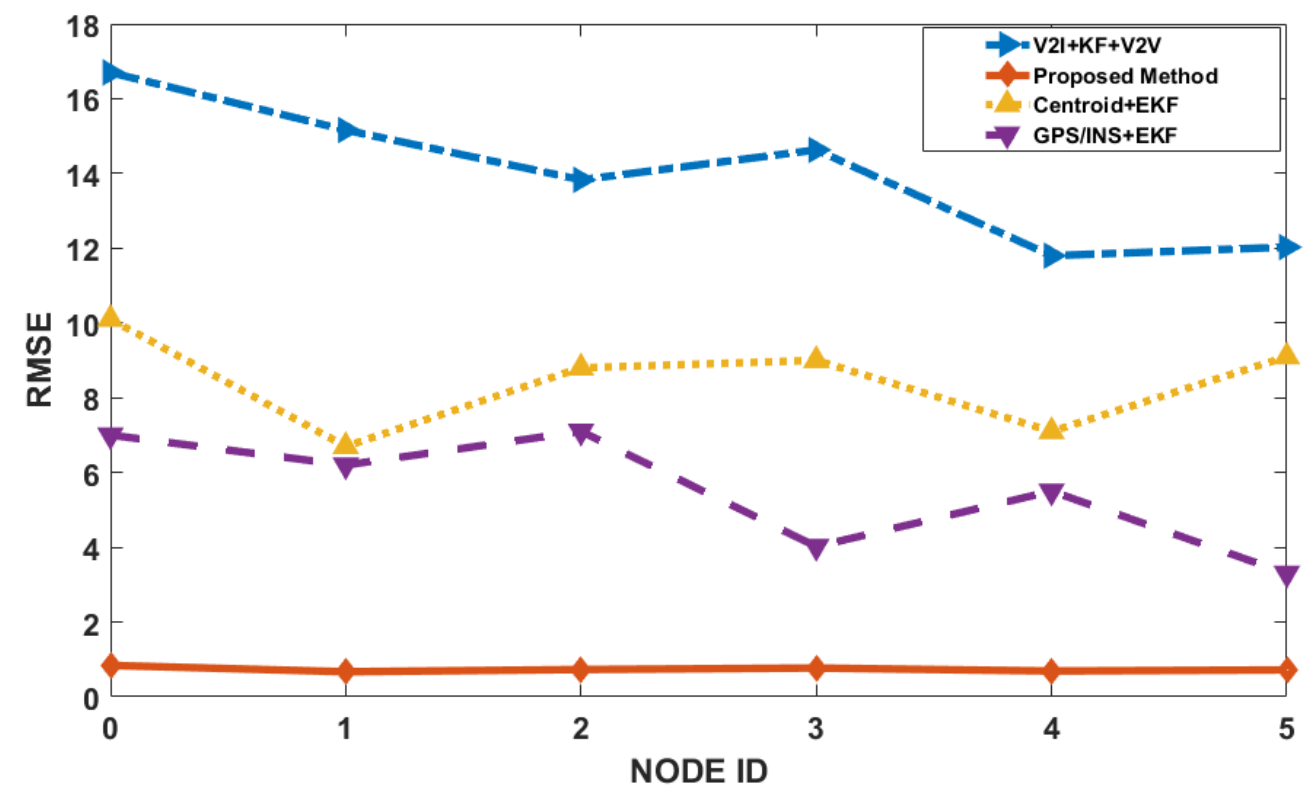

Figure 4. Root mean square error for a distance error of $0.5 \mathrm{~m}$

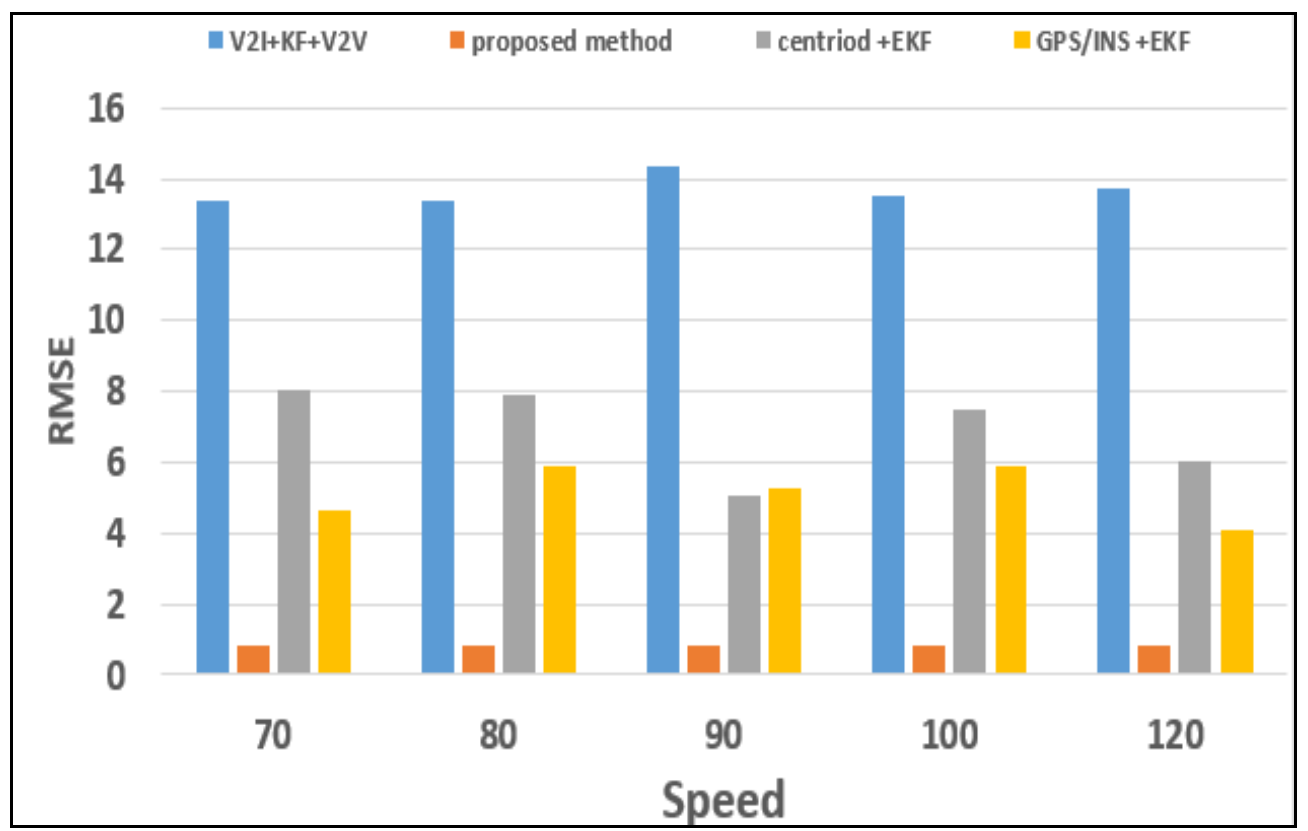

Figure 5. Root mean square error at different velocities and 0.5 distance errors 


\subsection{Scenario 2}

The KarradaIn scenario involved a more complex dataset where vehicles traveled in different directions and entered at different points; there were also large differences between the coordinate systems for each road. This second scenario was used to investigate the ability of the EKF to reduce the accumulated errors and to determine its sensitivity to the adapted noise covariance matrix. Different speeds and different sizes of error in the distance measurements were used in the experiment. Again, the position accuracy was measured in terms of the RMSE. One RSU was sited at position $(1000,3000)$ and one at $(700,3000)$ in proposed method. The width of the road was $300 \mathrm{~m}$ larger than that used in the first scenario. The noise had a Gaussian distribution with a mean of 0 and a variance of $\sigma^{2}$. Figure 6 shows the estimated RMSE for a scenario involving six vehicles and a distance error of $0.5 \mathrm{~m}$. It can be seen that, for the proposed method, the RMSE is almost the same at all of the nodes. The application of the general intersection formula produces small errors at large road width. The RMSEs for the GPS/INS + EKF method are greater than for the centroid + EKF method. Again, this is due to the uncertainty in the angles and speeds measured by the INS, as an INS is only accurate over short periods. The results for the centroid +EKF method demonstrate the advantages of applying the similar triangle rules to find more neighbors that have a linear relationship with the target vehicle. Figure 7 shows the RMSE results obtained for different speeds, also for a distance error of $0.5 \mathrm{~m}$. In the case of the proposed method, increasing the speed does not appear to affect the accuracy of the position; again, this is because of the accuracy of the initial vehicle positions and the use of the virtual RSU estimation method. The greatest error in the position-nearly $1 \mathrm{~m}$ - occurs at a speed of 80 $\mathrm{km} / \mathrm{h}$, which again is because of the lower accumulated errors that result from the use of a virtual RSU and the increase in the virtual road width. At higher speeds, the GPS/INS+EKF method produces smaller errors than the centroid $+\mathrm{EKF}$ method, which increases the probability of finding neighbors moving in the same direction. The weighted centroid method is more affected by the weight factors. Figure 8 illustrates the effect of uncertainties in the measurements on the position accuracy. For all of the cooperative localization methods, the RMSE increases as the error in the distance increases. This demonstrates the need for using accurate range measurement techniques, accurate initial position and of the adjusted noise covariance matrix in the EKF.

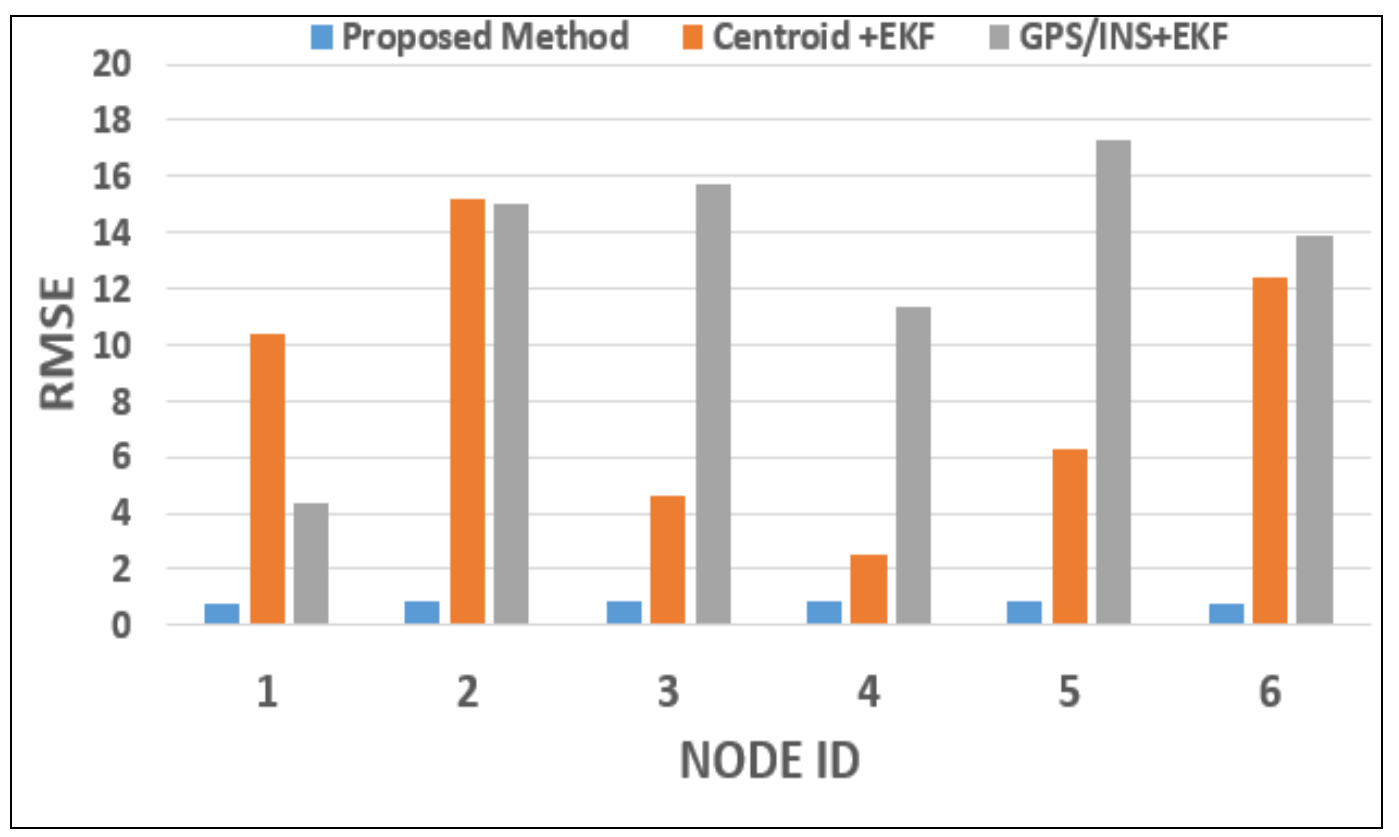

Figure 6. Root mean square error for a distance error of $0.5 \mathrm{~m}$ 
International Journal of Computer Networks \& Communications (IJCNC) Vol.13, No.6, November 2021

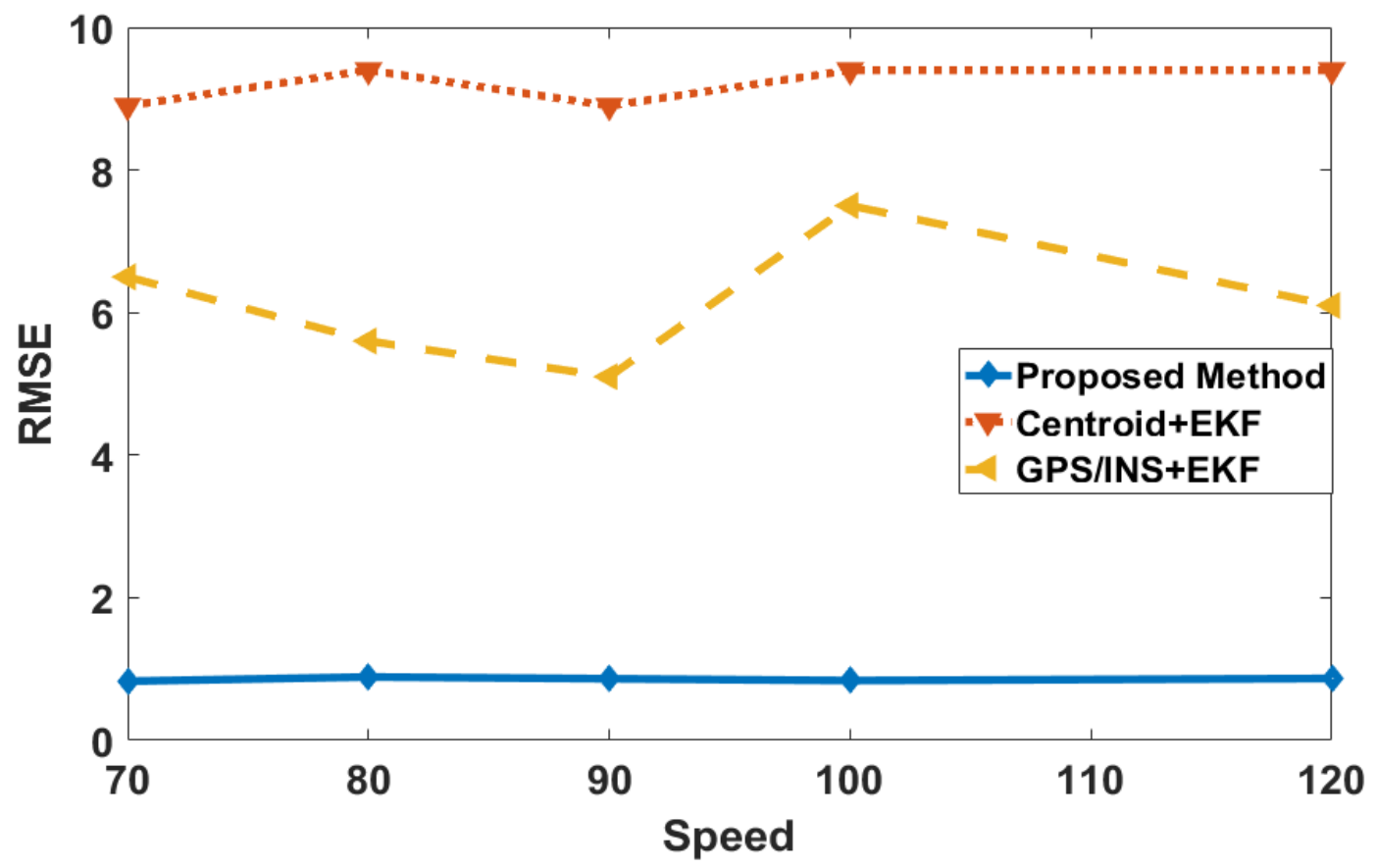

Figure 7. Root mean square error at different speeds for a distance error of $0.5 \mathrm{~m}$

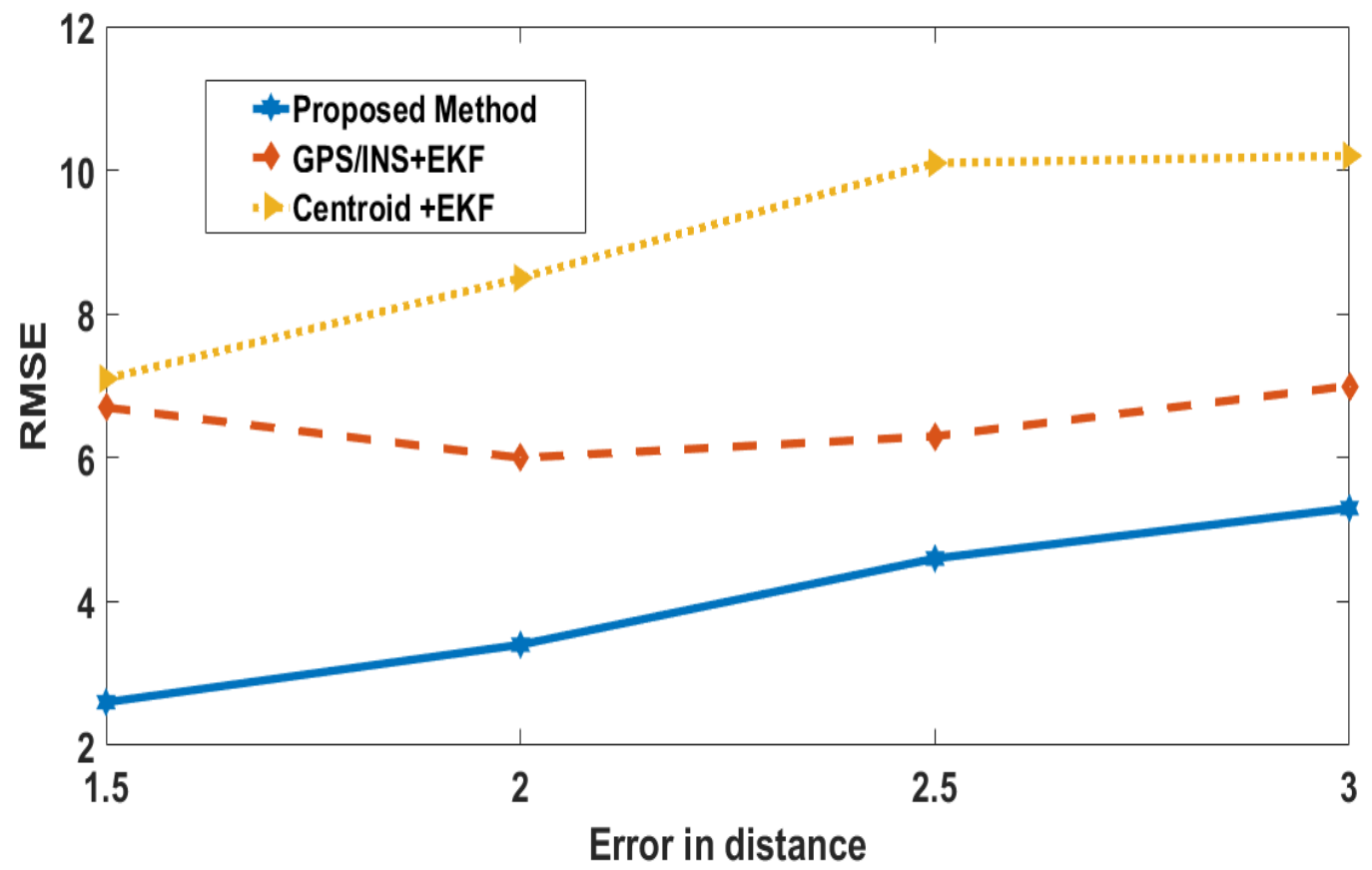

Figure 8. Root mean square error for different sizes of the distance error and a speed of $90 \mathrm{~km} / \mathrm{h}$

\section{DISCUSSION}

The nonlinear nature of localization and measurement uncertainty increase the difficulty of obtaining accurate vehicle positions. The relative position methods are based on communication 
between a vehicle and RSUs or with neighboring vehicles, is more affected by signal noise and the errors are accumulated over time. Localization in V2I communication perform better than $\mathrm{V} 2 \mathrm{~V}$ communication; the cost of the installed large number of RSU is high. V2V communication does not involve any costs but requires very accurate measurements. In the GPS/INS + EKF method [21], the coupled of GPS and INS systems produces positive results over short periods with some small errors. These errors are accumulated over time. The cost of attached inertial navigation frame affect the accuracy degree in measurements. EKF algorithms are sensitive to the noise covariance matrix and initial states. The method proposed in this paper uses fixed RSUs to help predict errors in the distance measurements. The initial vehicle positions are estimated using a general formula for the intersection of two circles and this gives more accurate results than updates derived using a kinematic model. In addition, the use of this general intersection formula allows different vehicle mobility models to be studied without affecting the position accuracy. The failure of an RSU affects the availability of position estimates and increases uncertainty in the measurements. Using a virtual RSU based on motion parameters has a positive impact on the position accuracy and increases the virtual road width. The application of the general intersection formula is more effective where the road is wide. In addition, the intersection method depends on the distance and, therefore, the angle has less impact on the accuracy being limited to determining whether the signs of the two coordinates are positive or negative. In the V2I + KF + V2V method [10], the use of two different communication methods does not appear to reduce the accumulation errors; again, this is because of the range measurement techniques used and the application of the $\mathrm{KF}$ algorithm. The KF algorithm is more applicable to linear problems. The centroid $+\mathrm{EKF}$ method can also be used to estimate vehicle positions. In [25], the rules for similar triangles were used to find neighboring vehicles with linear relations. However, this is difficult to do because of the lane changes made by vehicles. The use of fixed weights reduces the position accuracy due to errors and noise in the measurements. Highly accurate range measurement techniques should be used that represent high-energy consumption. Attempts are being made to organize the types of routing communication used in vehicular networks to decrease the uncertainty in measurements. Weight factors should give a good indicator of closeness degree for every neighboring vehicles and that can be used as a measure of uncertainty in measurements. This will have the benefit of increasing the degree of certainty in measurements as the dependence on $\mathrm{V} 2 \mathrm{~V}$ communication systems increases because of attempts to reduce the costs associated with building more RSUs.

\section{CONClusion}

In this paper, a cooperative localization method for improving position accuracy based on V2I communication and distance information was introduced. The proposed method consists of three steps. First, V2I communication is used to send two messages to each vehicle and to estimate the distance to each vehicle. Second, good estimates of the vehicle positions are obtained by applying the general equation for the intersection of two circles. These estimates are further improved by applying an EKF to reduce the uncertainty in the measurements by linearizing the nonlinear distance function. In case of the failure of one RSU, a virtual RSU is estimated so that estimates of the vehicle positions can continue to be made. The experimental results showed that the proposed method was the most efficient of the methods that were tested. The performance is approached to $1 \mathrm{~m}$ approximately. The use of GPS/INS, the cosine rule method and weighted centroid method did not produce good estimates of the initial positions. Data fusion algorithms were found to be more sensitive to the initial parameters and noise covariance matrix.

Future works, hybrid communication approach mixes the benefits of using two types of communications as a way to reduce accumulation error and substitute the high cost deployment of more roadside units along roads. Usage of high accurate range methods increases certainty degree of measurements. The multi-hop or clustering routing protocols helps to find neighbour with high accurate measurement. 
International Journal of Computer Networks \& Communications (IJCNC) Vol.13, No.6, November 2021

\section{CONFLICTS OF INTEREST}

The author declares no conflict of interest

\section{REFERENCES}

[1] Mengying Ren, Lyes Khoukhi, Houda Labiod, Jun Zhang, Véronique Vèque. (2017). A mobilitybased scheme for dynamic clustering in vehicular ad-hoc networks (VANETs). International Journal of Vehicular communications, Vol. 9, 233-241.

[2] Mathieu Nguyen-H and Chi Zhou. (2010). Improving GPS/INS Integration through Neural Networks", International Journal of telecommunications, Vol. 2, NO 2, 1-6.

[3] Azzedine Boukerche, Horacio A.B.F. Oliveira, Eduardo F. Nakamura, Antonio A.F. Loureiro. (2008). Vehicular Ad Hoc Networks: A New Challenge for Localization-Based Systems. International Journal of Computer communications, Vol. 31, No. 12, 2838-2849.

[4] Muhammad Abugabal, Yasmine Fahmy and Hazim Tawfik. (2021). NOVEL position estimation using differential timing information for asynchronous LTE/NR networks, International Journal of Computer Networks \& Communications (IJCNC), vol 13, No 4,39 -52.

[5] Zineb Squalli Houssaini, Imane Zaimi, Maroua Drissi and Mohammed Oumsis. (2018). Trade-off between accuracy, cost, and QoS using a beacon-on-demand strategy and Kalman filtering over a VANET. International Journal of Digital Communications and Networks, Vol. 4, No 1, 13-26.

[6] Ming-Fong Tsai, Po-Ching Wang, Ce-Kuen Shieh, Wen-Shyang Hwang, Naveen Chilamkurti, Seungmin Rho, Yang Sun Lee. (2015). Improving positioning accuracy for VANET in real city environments. International Journal of of Supercomputing, Vol. 71, No 6, 1975-1995.

[7] Umar Iqbal, Aime Francis Okou Aboelmagd Noureldin. (2008). An Integrated Reduced Inertial Sensor System-RISS/GPS for Land Vehicles. In: Proc. of International Conf on Position, Location and Navigation Symposium, Monterey, CA, USA, 1014-1021.

[8] Matthew Cossaboom, Jacques Georgy, Tashfeen Karamat, and Aboelmagd Noureldin. (2011). Augmented Kalman Filter and Map Matching for 3D RISS/GPS Integration for Land Vehicles. International Journal of Navigation and Observation, Vol 2012, 1-16.

[9] Walaa Afifi, Hesham Hefny, Nagy Ramadan Darwish and Imane Fahmy. (2020). Relative Position Estimation in Vehicle Ad-Hoc Network. IoT and Cloud Computing Advancements in Vehicular AdHoc Networks, IGI Global, 48-83.

[10] Mohamad El-Cheikh Ali, Hassan Artail, Youssef Nasser. (2017) A Cooperative and Roadside Unitaided Localization Scheme for Vehicular Ad hoc Networks. In: Proc. Of Conf on Electrical and Computing Technologies and Applications, Ras Al Khaimah, United Arab Emirates, 1-5.

[11] Simon Haykin. (2001). Kalman Filtering and Neural Networks. John Wiley and Sons.

[12] Greg Welch and Gary Bishop. (2006). An introduction to the Kalman Filter. UNC Chapel Hill, TR 95-041, 1-16.

[13] A. Khitwongwattana and T. Maneewarn. (2008). Extended Kalman Filter with Adaptive Measurement Noise Characteristics for Position Estimation of an Autonomous Vehicle. IEEE/ASME International Conference on Mechtronic and Embedded Systems and Applications, 505-509.

[14] John Vince. (2005) Geometry for Computer Graphics. Springer-Verlag, London.

[15] Sugang Ma, Fuxi Wen and Zhongmin Wan. (2018). An Efficient GPS-Free Vehicle Localization Algorithm Using Single Roadside Unit and Receiver. In: Proc. Of Conf on networking and network applications, Xi'an, China, 310-313.

[16] Alessio Fascista, Giovanni Ciccarese, Angelo Coluccia; Giuseppe Ricci. (2017). A localization algorithm based on V2I communications and AOA estimation. International Journal of IEEE signal processing letters, Vol 24, No 1, 126 - 130.

[17] Schmidt, R.O. (1986). Multiple Emitter Location and Signal Parameter Estimation," International Journal of IEEE Transactions on Antennas and Propagation, Vol. 34, No. 3, 276-280.

[18] Himan Zarza, Saleh Yousefi and Abderrahim Benslimane. (2015). RIALS: RSU/INS-aided localization system for GPS-challenged road segments. International Journal of wireless communications and mobile computing, vol. 16, No. 10, 1290-1305.

[19] Chia -Ho-Ou. (2012). A roadside unit - based localization schema for vehicular ad hoc networks. International Journal of communication systems, Vol. 27, No. 1, 135-150. 
International Journal of Computer Networks \& Communications (IJCNC) Vol.13, No.6, November 2021

[20] Pinar Oguz-Ekim, Khaled Ali1, Zahra Madadi, Francois Quitin and Wee Peng Tay. (2016). Proof of Concept Study Using DSRC, IMU and Map Fusion for Vehicle Localization in GNSS-Denied Environments. In: Proc. Of Conf on on Intelligent Transportation Systems, Rio de Janeiro, Brazil, 841-846.

[21] Liping Du, Longji Chen, Xiaotian Hou, Yueyun Chen. (2019). Cooperative Vehicle Localization Base on Extended Kalman Filter in Intelligent Transportation System. In: Proc. Of Conf on Wireless and Optical Communication, Beijing, China, 1-5.

[22] Drawil, N.M. and Basir, O. (2010). Intervehicle-communication-assisted localization," International Journal of IEEE Transactions on Intelligent Transportation Systems, Vol. 11, No. 3, 678-691.

[23] Nabil Drawil, Otman Basir. (2010). Toward Increasing the Localization Accuracy of Vehicles in VANET. In: Proc. Of Conf on Vehicular Electronics and Safety, Pune, India, 13-18.

[24] Farhan Ahamed, Javid Taheri, Albert Y. Zomaya and Max Ot. (2012). VLOCI: Using Distance Measurements to Improve the Accuracy of Location Coordinates in GPS-Equipped VANETs. In: Proc. Of Conf on Mobile and Ubiquitous Systems: Computing, Networking, and Services, Vol 73, 149-161.

[25] Felipe Lobo, Danilo Grael, Horacio Oliveira, Leandro Villas, Abdulaziz Almehmadi and Khalil ElKhatib. (2019). Cooperative Localization Improvement Using Distance Information in Vehicular Ad Hoc Networks. International journal of sensors, Vol. 19, No. 23, 1-27.

[26] Hanan H. AL Malki, Abdellatif I Moustafa, Mohammed H. Sinky. (2021). An Improving position method using Extended Kalman filter. In: Proc. Of international learning \& technology conference, $182,28-37$.

[27] Aboelmagd Noureldin, Tash feen B. Karamat, Jacques Georgy. (2013). Fundamentals of Inertial Navigation, Satellite-Based Positioning and Their Integration. Springer-Verlag, Berlin Heidelberg.

[28] OMNET ++ web site: https://omnetpp.org/ intro/.

[29] Vehicular network simulation framework (VEINS): veins.car2x.org/documentation/.

[30] Simulation of urban mobility (SUMO): https://sumo.dlr.de/docs/.

[31] Chantakarn Pholpol, Teerapat Sanguankotchakorn.(2021). Traffic Congestion Prediction using Deep Reinforcement Learning in Vehicular Ad-hoc Networks (vanets). International Journal of Computer Networks \& Communications (IJCNC) Vol.13, No.4, 1-19. 